\title{
Lenguaje: instrumento del desarrollo humano
}

\author{
Aurora Martínez Romero, José Luis Ortega Sánchez y José de Jesús Alba Romero
}

\begin{abstract}
Resumen
El lenguaje es la capacidad propia del ser humano para expresar pensamientos y sentimientos por medio de la palabra. Los objetivos de este artículo son conocer el lenguaje como un instrumento del desarrollo humano y aplicar esto en el proceso de enseñanza-aprendizaje. De esta manera, se abordan diferentes aspectos del lenguaje, como su relación con el cerebro, la infancia, el desarrollo social y humano, el aprendizaje y la tecnología.
\end{abstract}

Palabras clave: zona del lenguaje, desarrollo del lenguaje, artivismo, enseñanza- aprendizaje, inteligencia emocional, procesamiento del lenguaje natural.

\section{LANGUAGE: AN INSTRUMENT OF HUMAN DEVELOPMENT}

\begin{abstract}
Language is the ability of human beings to express thoughts and feelings through words. The objectives of this article are the understanding of language as an instrument of human development and apply this in the teaching-learning process. In this way, different aspects of language are addressed, such as its relationship with the brain, childhood, social and human development, learning and technology.
\end{abstract}

Keywords: language zone, language development, artivism, teaching-learning, emotional intelligence, natural language processing. 
"Lenguaje: instrumento del desarrollo humano" Aurora Martínez Romero, José Luis Ortega Sánchez y José de Jesús Alba Romero Vol. 22, Núm. 5, septiembre-octubre 2021 Revista Digital Universitaria

\section{Aurora Martínez Romero}

aurora.martinez@ujed.mx

orcid.org/0000-0002-9894-504X

Sitio: Aurora Martínez Romero

Química Farmacéutica Bióloga especialidad en Análisis Bioquímico Clínicos. Es egresada de la Facultad de Estudios Superiores Cuautitlán, de la Universidad Nacional Autónoma de México (UnAm). Tiene una Maestría en Desarrollo y Procesamiento de Alimentos por la Facultad de Ciencias Químicas de la Universidad Juárez del Estado de Durango (UJED) y una Maestría en Bioquímica Clínica por la misma casa de estudios. También cuenta con un Doctorado en Ciencias Agropecuarias por la Universidad Autónoma Agraria Antonio Narro de Torreón, Coahuila. Participan en la formación de recursos humanos, mediante la dirección de tesis de pregrado y posgrado con proyectos justificables con impacto académico y científico, de donde han emanado artículos publicados en revistas nacionales e internacionales, arbitrados e indexados, libros como autor y coautor, así como publicaciones en memorias de congreso regional, nacional e internacional. Es profesora investigadora de tiempo completo adscrita a la División de Estudios de Posgrado e Investigación de la Facultad de Ciencias Químicas ujeD. También ha sido Coordinadora de Posgrado e Investigación (2012-2018), y Coordinadora General de la Maestría en Ciencias Químicas y Coordinadora Interna del Doctorado en Biomedicina. Es además miembro del Sistema Nacional de Investigadores sN Nivel 1 (20142016), miembro del Registro CONACYT en Evaluadores Acreditados (RCEA) en el área 3 Medicina y Salud, miembro del Sistema Estatal de Investigadores SEl Nivel 1 (2021-2023) y miembro del Programa para el Desarrollo Profesional Docente, para el tipo superior PRODEP (2021-2024). Es responsable del Cuerpo académico de Bacteriología Médica Diagnóstica y Salud Pública en consolidación.

José de Jesús Alba Romero

josedejesusalba@ujed.mx orcid.org/0000-0003-2324-3251

Facebook: @Jesús Alba Romero

Químico Farmacéutico Biólogo con especialidad en Microbiología. Es egresado de la Facultad de Ciencias Químicas de la Universidad Autónoma de Coahuila unidad Saltillo. Tiene una Maestría en Salud Pública por la Facultad de Medicina de la ujed y una Maestría en Bioquímica Clínica por la Facultad de Ciencias Químicas de la misma casa de estudios, además de un Doctorado en Ciencias Biomédicas también por la ujed. Trabaja en la dirección de tesis de licenciatura con diversos proyectos, publicados en congresos y en revistas arbitradas e indexadas, regionales, nacionales e internacionales. Es además profesor investigador de tiempo completo adscrito a la División de Estudios de Posgrado e Investigación Facultad de Ciencias Químicas uJed. Está certificado por la Federación Nacional de Químicos Clínicos A.C. ConAQuic, por el Colegio Nacional de Químicos Farmacéuticos Biólogos de México y por el Consejo de Certificación Profesional de las Ciencias Químico Farmacéuticas. Es miembro del Programa para el Desarrollo Profesional Docente, para el tipo superior PRODEP (2021-2024) y miembro del Cuerpo académico Bacteriología Médica Diagnóstica y Salud Pública en consolidación. También es profesor de cursos de capacitación en bacteriología y control de calidad.

\section{José Luis Ortega Sánchez}

joeortega899@gmail.com

Médico Veterinario Zootecnista egresado de la Facultad de Veterinaria de la unam. Tiene una Maestría en Salud Pública por la Facultad de Medicina de la ujed y un Doctorado en Educación por la Universidad Autónoma de Coahuila. Asimismo, lleva a cabo la dirección de tesis de licenciatura. También es profesor investigador de tiempo completo adscrito al Departamento de Zonas Áridas en la División de Sistemas Pecuarios de la Unidad Regional Universitaria de Zonas Áridas de la Universidad Autónoma Chapingo. 
"Lenguaje: instrumento del desarrollo humano"

Aurora Martínez Romero, José Luis Ortega Sánchez y José de Jesús Alba Romero

Vol. 22, Núm. 5, septiembre-octubre 2021

Revista Digital Universitaria

\section{Introducción}

El lenguaje es la capacidad propia del ser humano para expresar pensamientos y sentimientos por medio de la palabra. Asimismo, es un sistema de signos que utiliza una comunidad para comunicarse oralmente o por escrito. Identifica además al hombre desde la perspectiva cultural, para que se sienta parte sustancial del lugar que ocupa en el universo. Por lo tanto, el lenguaje es parte de nuestra identidad, que se ve reflejada en nuestra cultura, tradiciones, historias, raíces comunes, ideales, valores, costumbres, que nos diferencia de cualquier otro ser humano, lo que nos hace ser únicos e irrepetibles, y tener un sentido de nacionalidad y pertenencia.

Además, el conocimiento del lenguaje facilita la comprensión del universo, saber identificar un problema y tomar medidas lógicas para encontrar una solución. Esto permite entender las emociones, lo que lo convierte en el vehículo fundamental para la buena interacción social y el desarrollo de la afectividad. En la niñez, el lenguaje es el instrumento que conlleva a un aprendizaje escolar satisfactorio, fundamental para el logro de un aprendizaje significativo y así afianzar el conocimiento posterior. Por ello, los objetivos de este artículo son conocer el lenguaje como instrumento del desarrollo humano y aplicar su conocimiento en el proceso enseñanza-aprendizaje.

\section{El lenguaje y el cerebro}

El lenguaje está en constante interacción con procesos neuronales, por lo que juega un papel central en el cerebro humano: desde cómo se procesa el color hasta cómo se valora un juicio moral o un acto mental que permite diferenciar entre lo correcto o incorrecto. González y Hornauer-Hughes (2014) determinan que las funciones cerebrales superiores, tales como el lenguaje, son un sistema funcional que depende del trabajo integrado de todo un grupo de zonas corticales y subcorticales, cada una de las cuales aporta su propia contribución al resultado final (p.143).

Fura 1. Cara lateral izquierda del cerebro. Áreas corticales y tractos relacionados con el lenguaje. B. Área de Broca. W. Área de Wernicke. SM. Circunvolución supramarginal. A. Circunvolución angular. FE. Función ejecutiva. PS. Procesamiento sintáctico. F. Fusiforme. M. Semántica. Memoria semántica. FLS-II. Fascículo longitudinal superior $2^{\circ}$ componente. FLS-III. Fascículo longitudinal superior 3. ${ }^{\text {er }}$ componente. FLS-IV (FA). Fascículo longitudinal superior $4{ }^{\circ}$ componente (fascículo arqueado). FLS-V. Fascículo longitudinal superior 5. $^{\circ}$ componente. FU. Fascículo uncinado. FLI. Fascículo longitudinal inferior. FFOl. Fascículo fronto-occipital inferior (González y Hornauer-Hughes 2014).

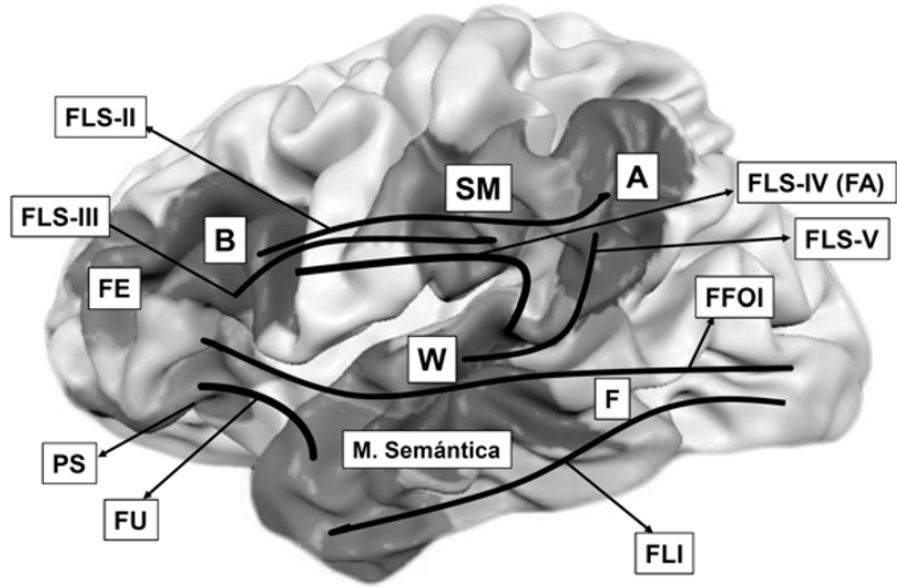

En Chile, González y Hornauer-Hughes (2014) analizaron las bases neurales del lenguaje como la relación entre el cerebro y el lenguaje, que describen las diferentes áreas y tractos cerebrales que participan en el sistema funcional del lenguaje (p.143). Localizaciones que corresponden a un sujeto diestro, adulto, que sabe leer y escribir (ver figura 1). 
"Lenguaje: instrumento del desarrollo humano" Aurora Martínez Romero, José Luis Ortega Sánchez y José de Jesús Alba Romero Vol. 22, Núm. 5, septiembre-octubre 2021

Revista Digital Universitaria

González y Hornauer-Hughes concluyen que la lateralización del lenguaje depende de una serie de variables tales como la dominancia manual, edad, sexo y escolaridad. Las principales áreas cerebrales relacionadas con el lenguaje se encuentran en el hemisferio izquierdo en la región perisilviana. Éstas son las áreas de Broca, Wernicke, circunvoluciones supramarginal y angular; ínsula anterior, el polo y las circunvoluciones segunda y tercera de ambos lóbulos temporales. Estas áreas están unidas a través de tractos, como el fascículo arqueado que forma parte de la vía dorsal del lenguaje que está relacionada con la expresión. Otros tractos son el uncinado, fascículo fronto-occipital y longitudinal inferior que forman parte de la vía ventral del lenguaje que está relacionada con la comprensión. Cada una de estas áreas son puntos de convergencia, las cuales se relacionan con múltiples regiones del cerebro, formando una extensa red neuronal (p.148).

Ardila y colaboradores (2016) en una investigación realizada en Miami, Estados Unidos, partieron de los estudios contemporáneos de imágenes cerebrales, para reanalizar la localización y extensión del área del lenguaje con relación a las diferentes áreas de Brodmann. Propusieron que existe una zona del lenguaje en el cerebro que corresponde, en general, a la región perisilviana del hemisferio izquierdo, y que incluye el área de Broca (tercera circunvolución frontal) y el área de Wernicke (el segmento posterior de la primera circunvolución del lóbulo temporal), y un centro del lenguaje escrito (la circunvolución angular). Se podría suponer que la ínsula representa una región central en el procesamiento lingüístico y se relaciona no sólo con las funciones de producción verbal, sino también con la comprensión del lenguaje. En ese sentido, se puede mantener la hipótesis de que la ínsula es, en verdad, una estación central relacionada con la coordinación entre los dos sistemas lingüísticos cerebrales: léxicosemántico (temporal) y gramatical (frontal).

\section{El lenguaje y la infancia}

Barbosa y colaboradores (2018) investigaron el desarrollo de las funciones ejecutivas y su relación con el lenguaje oral, así como las habilidades iniciales de alfabetización y el comportamiento en niños preescolares (p.121). Dentro del desarrollo infantil es importante analizar las funciones ejecutivas, lo que consiste en pruebas computarizadas para preescolares, porque se logra un desarrollo más integral. Se discuten tres marcadores destacados del control: el lenguaje proactivo, efecto de idioma dominante; los costos de mezcla de idiomas, y el efecto del bloqueo orden-idioma. Con base en estos tres aspectos, se puede implementar un control del lenguaje proactivo -o sea, el proceso del idioma implementado como una anticipación a cualquier interrupción para el cual se seleccionan las palabras óptimas del idioma materno-, para restringir principalmente la interferencia del primer lenguaje durante la producción de lenguaje bilingüe, pero generalmente está ausente durante la comprensión de este último (Declerck, 2019, p. 1). 
"Lenguaje: instrumento del desarrollo humano" Aurora Martínez Romero, José Luis Ortega Sánchez y José de Jesús Alba Romero Vol. 22, Núm. 5, septiembre-octubre 2021 Revista Digital Universitaria

En todo esto hay un control socioemocional que es oportuno, claro, preciso y concreto, y el cual se da además con moderación, cortesía y respeto, logrando así una comunicación efectiva y cálida. La función cognitiva influye directamente en el estado cognitivo de los adultos mayores, en cuanto a funciones ejecutivas y comprensión del lenguaje (Delgado-Lozada et al., 2019, p. 103891).

Asimismo, después de un accidente cerebrovascular infantil grave se evaluó el lenguaje y los resultados cognitivos, en donde el lenguaje y el coeficiente intelectual verbal fueron significativamente más bajos $(p<0.01)$ entre los pacientes con lesiones en el hemisferio izquierdo en oposición al derecho. Después de una lesión en el hemisferio izquierdo, las habilidades del lenguaje no fueron asociadas con la edad sino al accidente cerebrovascular, pero para las lesiones del hemisferio derecho, el lenguaje era más deteriorado entre los niños que eran más jóvenes al inicio del accidente cerebrovascular (de Montferrand et al., 2019, p. 509).

\section{El lenguaje y el desarrollo social y humano}

Dove (2019) dice que "el lenguaje influye en la cognición social, las palabras como herramientas sociales: lenguaje, socialidad y fundamento interno en conceptos abstractos son herramientas para dar forma al estado interno de nuestras mentes/cerebros" (p. 1). Lo anterior es consistente con el papel de los eventos y las memorias episódicas en el procesamiento de los conceptos abstractos, enlazando con la importancia de la cognición social compuesta por palabras como herramientas sociales que presentan un conjunto rico de mecanismos, hipótesis y predicciones que serán investigadas y refinadas en los próximos años, y avanzarán en nuestra comprensión de los conceptos abstractos (Desai, 2019, p. 166).

En ese sentido, a finales de la década de los ochenta surge el concepto artivismo, combinación de arte y activismo. Es un nuevo lenguaje educativo que viene a ser un instrumento del desarrollo humano dentro de una sociedad cambiante y transformadora. Por consiguiente, se trata de un fenómeno de protesta de forma creativa.

El artivismo es un lenguaje actual de autonomía y libertad, es un fenómeno global de importancia creciente. Aunque sus antecedentes se remontan décadas atrás, la importancia que ha adquirido como lenguaje cercano a la vida social y a las nuevas generaciones es algo completamente novedoso. En tanto medio o lenguaje de transformación social, puede servir para dar nueva energía a las necesidades de expresión en las ciudades y entornos urbanos actuales. Su valor formativo y su capacidad para romper las fronteras de las aulas e implicar en sus prácticas a los jóvenes (Aladro-Vico et al., 2018, p. 11).

En el mismo orden de ideas, el lenguaje en la comunicación científica requiere del conocimiento en el área en que se necesite ser contextualizado, poniendo atención a las diferencias culturales, la transparencia y reproducibilidad de la investigación, siempre bajo la consideración de comunicar los nuevos 
"Lenguaje: instrumento del desarrollo humano"

Aurora Martínez Romero, José Luis Ortega Sánchez y José de Jesús Alba Romero

Vol. 22, Núm. 5, septiembre-octubre 2021

Revista Digital Universitaria

hallazgos (Dejesus et al., 2019, p. 18370). Recientemente, surge el concepto de "ciudad en un lenguaje de derechos, concepto que permite abrir nuevas líneas de investigación para explorar derechos civiles, políticos y sociales ampliamente aceptados y expresados en un lenguaje de derechos, como salud, educación, memoria o la identidad" (Benitez, 2019, p. 24).

\section{El lenguaje y el aprendizaje}

Bravo concibe:

La psicopedagogía del aprendizaje del lenguaje escrito, como un paradigma de investigación que integra a la neuropsicología, la psicología cognitiva y la lingüística con la educación. He aplicado en él, el término aprendizaje del lenguaje escrito, en vez de enseñar o aprender a leer y escribir. Esta modificación no es solamente semántica, sino epistemológica (2018, p. 2).

Al respecto, "las investigaciones sobre el aprendizaje de la lengua escrita y las dislexias nos muestran una historia de pensamiento científico, que culminó en las neurociencias de la educación" (Bravo, 2018, p. 4). Bajo este ámbito, la función del lenguaje en el psicoanálisis no busca representar la realidad o comunicar un sentido preexistente, sino de realizar la verdad del sujeto con certeza inherente al hecho de hablar (De la Maza, 2019, p. 37).

En el contexto de la formación de profesores en lenguaje y comunicación, Calderón propone instrumentos para validar ambientes didácticos de aprendizaje, representados por la acción de la teoría del diseño, mediante la evaluación y refinamiento de artefactos asociados con la investigación y como resultado de las relaciones que se tejen entre el ambiente problema y el campo de conocimiento (2018, p. 57).

De acuerdo con la satisfacción del aprendizaje, Alconero-Camarero y colaboradores (2017) analizaron la inteligencia emocional como un modelo transaccional cognitivo, que se basa en los procesos de pensamiento que intervienen para mediar el estrés y el entorno experimentados. La educación socioemocional ${ }^{1}$ favorece el desarrollo del potencial humano porque provee recursos internos para enfrentar dificultades a lo largo de la vida. Asimismo, los autores concluyen que la inteligencia emocional y la satisfacción del aprendizaje son cualidades deseables en los estudiantes, especialmente porque tienen un papel relevante en la satisfacción del propio aprendizaje (p. 100).

De la misma manera, de Stefani y de Marco (2019) enfatizan la hipótesis del lenguaje como "encarnado" en la experiencia sensorial y motora, lo cual ha sido ampliamente discutido en el campo de la neurociencia cognitiva, en donde el

${ }^{1}$ El aspecto socioemocional considera la retroalimentación efectiva y la comunicación cálida dependientes de las actitudes y aptitudes de los individuos. lenguaje se centra en estudios que investigan la relación funcional entre señales de comunicación entre el gesto y el habla, y los circuitos neuronales involucrados en su procesamiento y producción que expresan mensajes emocionales en la extensión del contexto de interacción social (p. 1). 
"Lenguaje: instrumento del desarrollo humano"

Aurora Martínez Romero, José Luis Ortega Sánchez y José de Jesús Alba Romero

Vol. 22, Núm. 5, septiembre-octubre 2021

Revista Digital Universitaria

Incluso, en la actualidad se ha enfatizado el conocimiento sobre la influencia del lenguaje (términos coloquiales) en la educación alimentaria en el uso de algunos términos usados en materia de alimentación como: comida chatarra, chucherías y aperitivos (Tejedor-Martín et al., 2019, p. 30). Así, la web semántica² nos permite hacer uso de vocabularios controlados (Tintinago et al., 2018, p. 528).

Al respecto, Miranda (2011) explica que:

mediante el lenguaje nos aproximamos a nosotros mismos y a los demás, reflexionamos sobre nuestro proceder, nuestras emociones y condiciones internas; a través de lenguaje comprendemos e interpretamos la naturaleza, el mundo de las estructuras físicas y formales; mediante el lenguaje heredamos conocimiento de las generaciones anteriores y trasmitimos ideas a las próximas, construimos imágenes de nuestro ser y el de los otros, elaboramos máquinas, artefactos y programas, pero, lo más fascinante, con el lenguaje indagamos sobre el mismo lenguaje y en su relación intrínseca con el pensamiento (p. 161).

\section{El lenguaje y la tecnología}

Recientemente, Tintinago y colaboradores (2018) propusieron que: "El campo de estudio que se centra en las interacciones entre el lenguaje humano y los ordenadores se llama procesamiento del lenguaje natural (PLN)" (p. 528). En éste convergen la informática, la inteligencia artificial, y la lingüística computacional. El PLN, explican, busca que los ordenadores puedan analizar, comprender y entender el lenguaje humano. Por ello, implementa un lenguaje natural para comunicarnos con la computadora de manera más entendible y natural, ya que permite entender las oraciones que le sean suministradas (p. 537).

\section{Conclusiones}

El lenguaje es un instrumento importante en el desarrollo humano, en particular en el proceso enseñanza-aprendizaje. El uso del PLN facilita la ejecución de programas que cumplan tareas relacionadas con el lenguaje. Los resultados del aprendizaje del lenguaje dependen de las características de las actividades educativas. La educación, la cultura y la investigación coadyuvan a la obtención de una formación integral y así alcanzar la libertad.

En términos de lo que antecede, se sugiere que el primer paso es liberarse de aspectos del lenguaje habitual para mejorarlo y por ende la comunicación humana, comprender su rol en el aprendizaje y como mediador del mismo. El lenguaje como instrumento del desarrollo humano se vincula con un proceso histórico físico y social de manera que se tiene que afrontar la construcción y adquisición del conocimiento de nuestro lenguaje que nos caracterizará y definirá dentro de lo que es la formación integral durante la existencia de cada individuo, lo que permitirá mejorar las relaciones interpersonales para tener mayor participación en las prácticas sociales y culturales logrando así, el éxito de las organizaciones.

\footnotetext{
${ }^{2}$ La semántica se refiere a los aspectos del significado, sentido o interpretación de signos lingüísticos como símbolos, palabras, expresiones o representaciones formales.
} 
"Lenguaje: instrumento del desarrollo humano"

Aurora Martínez Romero, José Luis Ortega Sánchez y José de Jesús Alba Romero

Vol. 22, Núm. 5, septiembre-octubre 2021

Revista Digital Universitaria

\section{Referencias}

* Aladro-Vico, E., Jivkova-Semova, D. y Bailey, O. (2018). Artivismo: Un nuevo lenguaje educativo para la acción social transformadora. Comunicar, xxvi(57), 8-18. https:// doi.org/10.3916/C57-2018-01

* Alconero-Camarero, A. R., Sarabia-Cobo, C. M., González-Gómez, S., IbáñezRementería, I., Lavín-Alconero, L. y Sarabia-Cobo, A. B. (2018). Nursing students' emotional intelligence, coping styles and learning satisfaction in clinically simulated palliative care scenarios: An observational study. Nurse Education Today, 61, 94100. https://doi.org/10.1016/j.nedt.2017.11.013

* Ardila, A., Bernal, B.y Rosselli, M. (2016). Área cerebral del lenguaje: una reconsideración funcional. Rev Neurol, 62, 97-106. https://doi.org/10.33588/rn.6203.2015286

* Barbosa, C., Martins, N., Lamarca, G. y Gotuzo, A. (2018). Executive functions in preschool children: development and relationships with language and behavior. Revista Psicologia: Teoria e Prática, 20, 121-137. http://dx.doi.org/10.5935/19806906/psicologia.v20n3p121-137

* Benitez, J. A. (2019). Los límites del derecho a la ciudad como prisma para entender conflictos urbanos: acción colectiva y lenguaje de derechos en la Ciudad Autónoma de Buenos Aires. Población \& Sociedad, 26, 6-31. http://dx.doi. org/10.19137/pys-2019-260102

- Bravo, L. (2018). El Paradigma de las Neurociencias de la Educación y el Aprendizaje del Lenguaje Escrito: Una Experiencia de 60 Años. PSYKHE, 27(1), 1-11. http://dx.doi. org/10.7764/psykhe.27.1.1101

* Calderón, D. I., Castañeda-Peña, H., Borja-Orozco, M. G., Quitián-Bernal, S. P. y Suárez-Reina, A. Y. (2018). Instrumentos para validar Ambientes Didácticos de Aprendizaje (ADA) para la formación de profesores de lenguaje y comunicación en y para la diversidad: una herramienta en la didáctica del lenguaje. Signo y Pensamiento, xxxvII, 56-76. https://doi.org/10.11144/Javeriana.syp37-72.ivda

* De la Maza, L. M. (2019). Hegel en Lacan. Las trampas de lo imaginario y la función del lenguaje en la construcción del sujeto. Veritas, 43, 29-47. http://dx.doi. org/10.4067/S0718-92732019000200029

* De Montferrand, C., Vassel-Hitier, J., Yvon-Chaou, E., Camara-Costa, H., Dellatolas, G. y Chevignard, M. (2019). Language and cognitive outcomes after childhood stroke: Theoretical implications for hemispheric specialization. Cortex, 120, 509523. https://doi.org/10.1016/j.cortex.2019.07.020

- De Stefani, E. y De Marco, D. (2019). Language, Gesture, and Emotional Communication: An Embodied View of Social Interaction. Frontiers in Psychology, 10, 1-8. https://doi.org/10.3389/fpsyg.2019.02063

* Declerck, M. (2019). What about proactive language control? Psychon Bull Rev, 1, 1-12. https://doi.org/10.3758/s13423-019-01654-1

* Dejesus, J. M., Callanan, M. A., Solis, G. y Gelman, S. A. (2019). Generic language in scientific communication. Proc Natl Acad Sci U S A, 116,18370-18377. https://doi. org/10.1073/pnas.1817706116 
"Lenguaje: instrumento del desarrollo humano" Aurora Martínez Romero, José Luis Ortega Sánchez y José de Jesús Alba Romero Vol. 22, Núm. 5, septiembre-octubre 2021 Revista Digital Universitaria

* Delgado-Losada, M. L., Rubio-Valdehita, S., Lopez-Higes, R., Rodríguez-Rojo, I. C., Prados Atienza, J. M., Garcia-Cid, S. y Montenegro, M. (2019). How cognitive reserve influences older adults' cognitive state, executive functions and language comprehension: A structural equation model. Arch Gerontol Geriatr, 84, 103891. https://doi.org/10.1016/j.archger.2019.05.016

* Desai, R. H. (2019). Access and content of abstract concepts: Comment on "Words as social tools: Language, sociality, and inner grounding in abstract concepts" by Anna M. Borghi et al. Phys Life Rev, 29,166-168. https://doi.org/10.1016/j. plrev.2019.03.010

* Dor, D. (2019). Language and innovation: Comment on "Replication and emergence in cultural transmission" by Monica Tamariz. Phys Life Rev, 30, 1-3. https://doi. org/10.1016/j.plrev.2019.08.016

* Dove, G. (2019). Language influences social cognition Comment on "Words as social tools: Language, sociality and inner grounding in abstract concepts" by Anna M. Borghi et al. Phys Life Rev, 29, 169-171. https://doi.org/10.1016/j.plrev.2019.03.007

* González, R. V., y Hornauer-Hughes, A. (2014). Cerebro y lenguaje. Rev Hosp Clin Univ Chile, 25, 143-153. https://cutt.ly/XWz26TZ

* Miranda Calderón, L. A. (2011). Lenguaje: Algo más que un mecanismo para la comunicación. Revista Electrónica Educare, xv, 161-170. https://cutt.ly/IWz9eBW

* Quesada-Perea, R. (2011). Impacto de las infotecnologías, la neurociencia y la neuroética en la educación. Revista Española de Pedagogía, LXIX, 289-303.

* Sandoval-Zuñiga, M. S., Figueroa-Olivares, C. R., Sepúlveda-Contreras, K. M. y Parada-Betancourt, J. E. (2018). Subcomponentes de la memoria operativa en niños con trastorno específico del lenguaje tipo mixto. Lenguas Modernas, 51, 4762. https://cutt.ly/6Wz9pW3

* Tejedor-Martín, F. J., Pérez-Llano, B., Mijancos-Gurruchaga, M. T. y GonzálezPanero, P. (2019). El lenguaje y la educación alimentaria. Resultados de un cuestionario poblacional. Revista Española de Comunicación en Salud, 10, 30-41. https://e-revistas.uc3m.es/index.php/RECS/article/view/4335

* Tintinago, A., Muñoz, Y., Uribe, G. A. y Álvarez-Sánchez, P. H. (2018). Etiquetado asistido de documentos de investigación mediante procesamiento de lenguaje natural y tecnologías de la web semántica. Scientia et Technica Año XXIII, 23(4) 528537. https://dialnet.unirioja.es/servlet/articulo?codigo=6809464

\section{Cómo CITAR ESTE ARTículo}

* Martínez Romero, Aurora, Ortega Sánchez, Jose Luis y Alba Romero, José de Jesús. (2021, septiembre-octubre). Lenguaje: instrumento del Desarrollo Humano. Revista Digital Universitaria (RDU), 22(5). http://doi.org/10.22201/ cuaieed.16076079e.2021.22.5.3 